\title{
MODELING OF MULTIMASS SYSTEMS TORSIONALLY DEFORMED WITH VARIABLE INERTIA
}

\author{
AMALIA PIELORZ AND MONIKA SKÓRA
}

Received 20 December 2005; Accepted 29 May 2006

Dynamic investigations of multimass discrete-continuous systems having variable moment of inertia are performed. The systems are torsionally deformed and consist of an arbitrary number of elastic elements connected by rigid bodies. The problem is nonlinear and it is linearized after appropriate transformations. It is shown that such problems can be investigated using the wave approach. Some analytical considerations and numerical calculations are done for a two-mass system with a special case of boundary conditions.

Copyright (C 2006 A. Pielorz and M. Skóra. This is an open access article distributed under the Creative Commons Attribution License, which permits unrestricted use, distribution, and reproduction in any medium, provided the original work is properly cited.

\section{Introduction}

The torsional vibrations of the systems with variable inertia usually are discussed using discrete models; see $[1,3,6,7]$. In [1] a two-mass discrete-continuous system is considered and the forced response of variable inertia is studied. From the technical literature it follows that variable inertia problems play an important role in dynamics of various mechanism and machines undergone torsional deformations, $[1,3,6,7]$.

In the present paper the results of [1] are generalized to multimass systems and the wave method is applied reducing appropriate problems for the solution of differential equations with a retarded argument. The derived equations are the base for appropriate numerical calculations. Some analytical considerations are presented for a two-mass system, according to [1], with a special case of boundary conditions.

\section{Governing equations for a multimass system}

Consider the discrete-continuous model of a system which consists of a suitable number of rigid bodies connected by means of shafts, Figure 2.1. The shafts are deformed torsionally and their central axes, together with elements settled on them, coincide with the main axis of the system. The $x$-axis is parallel to the main axis of the system, and its origin 


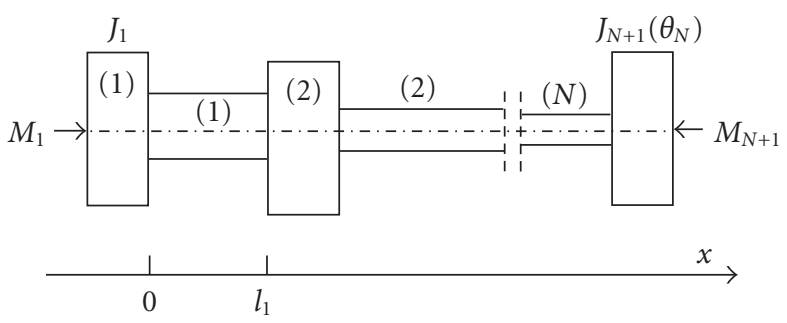

Figure 2.1. Discrete-continuous multimass torsional system.

coincides with the position of the left-hand end of the first shaft in an undisturbed state at time instant $t=0$.

The $i$ th shaft, $i=1,2, \ldots, N$, is characterized by length $l_{i}$, density $\rho$, shear modulus $G$, and polar moment of inertia $I_{0 i}$. The $i$ th rigid body of the model is characterized by the mass moment of inertia $J_{i}$. The mass moment of inertia of the last rigid body depends on the solution, that is, $J_{N+1}=J_{N+1}\left(\theta_{N}\right)$.

The rigid body $J_{1}$ represents an electrical motor and is loaded by the motor torque $M_{1}=M_{0}+K\left(\Omega_{0}-\partial \theta_{1} / \partial t\right)$, where $M_{0}$ is the nominal torque, $\Omega_{0}$ is the nominal angular velocity, and $K$ is the slope of the motor characteristic. In further considerations it is assumed that the motor operates at a constant angular velocity $\Omega_{0}$. The last rigid body is loaded by an equivalent resistance torque equal to $M_{N+1}=M_{0}$; see [1]. Damping in the system is neglected. We are interested in a forced response resulting from the variable inertia of $(N+1)$ th rigid body. For this reason, initial conditions are omitted.

Under the above assumptions, the determinations of displacements $\theta_{i}$ of the elastic elements of the considered system are reduced to solving $N$ classical wave equations

$$
\frac{\partial^{2} \theta_{i}}{\partial t^{2}}-c^{2} \frac{\partial^{2} \theta_{i}}{\partial x^{2}}=0, \quad i=1,2, \ldots, N
$$

with the following boundary conditions:

$$
\begin{gathered}
\frac{\partial \theta_{1}}{\partial t}=\Omega_{0} \quad \text { for } x=0 \\
\theta_{i}(x, t)=\theta_{i+1}(x, t) \quad \text { for } x=\sum_{k=1}^{i} l_{k}, i=1,2, \ldots, N-1, \\
-J_{i+1} \frac{\partial^{2} \theta_{i}}{\partial t^{2}}-G I_{0 i} \frac{\partial \theta_{i}}{\partial x}+G I_{0, i+1} \frac{\partial \theta_{i+1}}{\partial x}=0 \quad \text { for } x=\sum_{k=1}^{i} l_{k}, i=1,2, \ldots, N-1, \\
J_{N+1} \frac{\partial^{2} \theta_{N}}{\partial t^{2}}+\frac{1}{2} \frac{d J_{N+1}}{d \theta_{N}}\left(\frac{\partial \theta_{N}}{\partial t}\right)^{2}+G I_{0 N} \frac{\partial \theta_{N}}{\partial x}=-M_{N+1} \quad \text { for } x=\sum_{k=1}^{N} l_{k} .
\end{gathered}
$$

The condition in the cross-section $x=l_{1}+l_{2}+\cdots+l_{N}$ can have various forms. The aim of the paper, however, is to generalize some results given in [1], so this condition is taken 
in the form postulated in that paper. According to [1], this condition includes the nonlinear Eksergian equation.

The problem (2.1)-(2.2) is nonlinear. In order to obtain some effective solutions it is linearized. In order to linearize the last boundary condition in (2.2), new unknown functions are proposed:

$$
\theta_{i}(x, t)=\Omega_{0} t+\alpha_{i}(x, t)
$$

the variable mass moment of inertia $J_{N+1}\left(\theta_{N}\right)$ is expended in a Taylor series around $\Omega_{0} t$, and second- and higher-order terms are neglected. Besides, the static deformations corresponding to the nominal torque moment $M_{0}$ appearing in the first and the last conditions can be separated by introducing the following new transformations:

$$
\alpha_{i}(x, t)=\alpha_{i d}(x, t)-\frac{M_{0}}{G I_{0 i}}\left(x-\sum_{k=1}^{i} l_{k}\right)
$$

Then, after omitting the index $d$ for convenience, the problem (2.1)-(2.2) is reduced to solving $N$ equations

$$
\frac{\partial^{2} \alpha_{i}}{\partial t^{2}}-c^{2} \frac{\partial^{2} \alpha_{i}}{\partial x^{2}}=0, \quad i=1,2, \ldots, N
$$

with linear boundary conditions

$$
\begin{gathered}
\frac{\partial \alpha_{1}}{\partial t}=0 \quad \text { for } x=0 \\
\alpha_{i}(x, t)=\alpha_{i+1}(x, t)+\frac{M_{0}}{G I_{0, i+1}} l_{i+1} \quad \text { for } x=\sum_{k=1}^{i} l_{k}, i=1,2, \ldots, N-1, \\
-J_{i+1} \frac{\partial^{2} \alpha_{i}}{\partial t^{2}}-G I_{0 i} \frac{\partial \alpha_{i}}{\partial x}+G I_{0, i+1} \frac{\partial \alpha_{i+1}}{\partial x}=0 \quad \text { for } x=\sum_{k=1}^{i} l_{k}, i=1,2, \ldots, N-1, \\
J_{N+1}(t) \frac{\partial^{2} \alpha_{N}}{\partial t^{2}}+\frac{d J_{N+1}}{d t}(t) \frac{\partial \alpha_{N}}{\partial t}+G I_{0 N} \frac{\partial \alpha_{N}}{\partial x}+\frac{1}{2} \frac{d^{2} J_{N+1}}{d t^{2}}(t) \alpha_{N} \\
=-\frac{1}{2} \frac{d J_{N+1}}{d t}(t) \Omega_{0} \quad \text { for } x=\sum_{k=1}^{N} l_{k} .
\end{gathered}
$$

It is seen that though the first and the last conditions in (2.6) do not depend on $M_{0}$, this moment occurs in the relations for displacements in cross-sections where rigid bodies are located. However, when angular velocities and strains are considered, no effect of $M_{0}$ is observed. The same takes place in the case of a two-mass system with $N=1$. Moreover, it is seen now that the last boundary condition has such a form that a direct effect of variable inertia can be investigated. 
4 Modeling of systems with variable inertia

Upon the introduction of the following dimensionless quantities:

$$
\begin{gathered}
\bar{x}=\frac{x}{l_{1}}, \quad \bar{t}=\frac{c t}{l_{1}}, \quad \bar{\alpha}_{i}=\frac{\alpha_{i}}{\alpha_{0}}, \\
K_{r}=\frac{I_{01} \rho l_{1}}{J_{0}}, \quad E_{i}=\frac{J_{0}}{J_{i}}, \quad \bar{\Omega}_{0}=\frac{\Omega_{0} l_{1}}{\alpha_{0} c}, \\
\bar{M}_{0}=\frac{M_{0} l_{1}^{2}}{\left(J_{0} c^{2} \alpha_{0}\right)}, \quad \bar{J}_{N+1}(\bar{t})=\frac{J_{N+1}(t)}{J_{0}}, \quad \bar{l}_{i}=\frac{l_{i}}{l_{1}}, \quad B_{i}=\frac{I_{0 i}}{I_{01}},
\end{gathered}
$$

the determination of angular displacements $\alpha_{i}(x, t)$ is reduced to solving $N$ equations

$$
\frac{\partial^{2} \alpha_{i}}{\partial t^{2}}-\frac{\partial^{2} \alpha_{i}}{\partial x^{2}}=0, \quad i=1,2, \ldots, N
$$

with the following boundary conditions:

$$
\begin{gathered}
\frac{\partial \alpha_{1}}{\partial t}=0 \quad \text { for } x=0 \\
\alpha_{i}(x, t)=\alpha_{i+1}(x, t)+C_{i+1} \quad \text { for } x=\sum_{k=1}^{i} l_{k}, i=1,2, \ldots, N-1, \\
-\frac{\partial^{2} \alpha_{i}}{\partial t^{2}}-K_{r} B_{i} E_{i+1} \frac{\partial \alpha_{i}}{\partial x}+K_{r} B_{i+1} E_{i+1} \frac{\partial \alpha_{i+1}}{\partial x}=0 \quad \text { for } x=\sum_{k=1}^{i} l_{k}, i=1,2, \ldots, N-1, \\
J_{N+1}(t) \frac{\partial^{2} \alpha_{N}}{\partial t^{2}}+\frac{d J_{N+1}}{d t}(t) \frac{\partial \alpha_{N}}{\partial t}+K_{r} B_{N} \frac{\partial \alpha_{N}}{\partial x}+\frac{1}{2} \frac{d^{2} J_{N+1}}{d t^{2}}(t) \alpha_{N} \\
=-\frac{1}{2} \frac{d J_{N+1}}{d t}(t) \Omega_{0} \quad \text { for } x=\sum_{k=1}^{N} l_{k},
\end{gathered}
$$

where $\alpha_{0}$ is a fixed angular displacement, $C_{i}=M_{0}\left(K_{r} B_{i}\right)^{-1} l_{i}$, and the bars denoting dimensionless quantities are omitted for convenience.

The solutions of $(2.8)$, similarly to problems discussed in $[2,4,5]$, are sought in the form

$$
\alpha_{i}(x, t)=f_{i}\left(t-x+2 \sum_{k=1}^{i} l_{k}-\sum_{k=1}^{N} l_{k}\right)+g_{i}\left(t+x-\sum_{k=1}^{N} l_{k}\right), \quad i=1,2, \ldots, N .
$$

The functions $f_{i}$ and $g_{i}$ represent here the waves, caused by variable inertia, propagating in the $i$ th shaft in the positive and negative directions of the $x$-axis, respectively. They are continuous and equal to zero for negative arguments. In (2.10) it is taken into account that the first disturbance appears in the $i$ th element in the cross-section $x_{0 i}=l_{1}+l_{2}+$ $\cdots+l_{i}$ at time instant $t_{0 i}=l_{i+1}+l_{i+2}+\cdots+l_{N}$.

Substituting the solution (2.10) into the boundary conditions (2.9) and denoting the largest argument in each boundary condition separately by $z$, we obtain the following set 
of ordinary differential equations for unknown functions $f_{i}$ and $g_{i}$ :

$$
\begin{gathered}
f_{1}^{\prime}(z)=-g_{1}^{\prime}\left(z-2 l_{1}\right), \\
f_{i}(z)=f_{i-1}\left(z-2 l_{i}\right)+g_{i-1}\left(z-2 l_{i}\right)-g_{i}\left(z-2 l_{i}\right)-C_{i}, \quad i=2,3, \ldots, N, \\
r_{N 1} g_{N}^{\prime \prime}(z)+r_{N 2} g_{N}^{\prime}(z)+r_{N 3} g_{N}(z)=F(z)+r_{N 4} f_{N}^{\prime \prime}(z)+r_{N 5} f_{N}^{\prime}(z)+r_{N 6} f_{N}(z), \\
g_{i}^{\prime \prime}(z)+r_{i 1} g_{i}^{\prime}(z)=-f_{i}^{\prime \prime}(z)+r_{i 2} f_{i}^{\prime}(z)+r_{i 3} g_{i+1}^{\prime}(z), \quad i=N-1, N-2, \ldots, 1,
\end{gathered}
$$

where

$$
\begin{gathered}
r_{N 1}(z)=J_{N+1}(z), \quad r_{N 2}(z)=K_{r} B_{N}+J_{N+1}^{\prime}(z), \quad r_{N 3}(z)=\frac{J_{N+1}^{\prime \prime}(z)}{2} \\
r_{N 4}(z)=-r_{N 1}(z), \quad r_{N 5}(z)=K_{r} B_{N}-J_{N+1}^{\prime}(z), \quad r_{N 6}(z)=-r_{N 3}(z) \\
r_{i 1}=K_{r} E_{i+1}\left(B_{i}+B_{i+1}\right), \quad r_{i 2}=K_{r} E_{i+1}\left(B_{i}-B_{i+1}\right) \\
r_{i 3}=2 K_{r} E_{i+1} B_{i+1}, \quad F(z)=-\frac{J_{N+1}^{\prime}(z) \Omega_{0}}{2}
\end{gathered}
$$

In (2.11) coefficients are variable. They are solved numerically with zero initial conditions, similarly to equations derived in $[2,4,5]$.

\section{Analytical approach for $N=1$}

According to [1], the analytical approach can be used in the discussion of discretecontinuous systems with variable inertia when $N=1$. Then we have the single equation of motion:

$$
\frac{\partial^{2} \alpha_{1}}{\partial t^{2}}-\frac{\partial^{2} \alpha_{1}}{\partial x^{2}}=0
$$

and two boundary conditions:

$$
\begin{gathered}
\frac{\partial \alpha_{1}}{\partial t}=0 \quad \text { for } x=0 \\
J_{2}(t) \frac{\partial^{2} \alpha_{1}}{\partial t^{2}}+\frac{d J_{2}}{d t}(t) \frac{\partial \alpha_{1}}{\partial t}+K_{r} \frac{\partial \alpha_{1}}{\partial x}+\frac{1}{2} \frac{d^{2} J_{2}}{d t^{2}}(t) \alpha_{1}=-\frac{1}{2} \frac{d J_{2}}{d t}(t) \Omega_{0} \quad \text { for } x=1 .
\end{gathered}
$$

An arbitrary function $J_{2}(\theta)$ can be expressed in the form of a Fourier series,

$$
J_{2}(\theta)=\sum_{m=0}^{\infty}\left(a_{m} \cos m \theta+b_{m} \sin m \theta\right),
$$

where in our case $\theta=\Omega_{0} t$.

Upon substituting it to the boundary conditions, using a method of a small parameter $\varepsilon=a_{c} / a_{0}$, seeking the solution in the form

$$
\alpha_{1}(x, t)=\alpha_{10}(x, t)+\varepsilon \alpha_{11}(x, t)+\varepsilon^{2} \alpha_{12}(x, t)+\cdots,
$$


6 Modeling of systems with variable inertia

and collecting the terms of like power of $\varepsilon$, we obtain equations of motion:

$$
\frac{\partial^{2} \alpha_{1 i}}{\partial t^{2}}-\frac{\partial^{2} \alpha_{1 i}}{\partial x^{2}}=0, \quad i=0,1, \ldots
$$

with

$$
\begin{gathered}
\frac{\partial \alpha_{1 i}}{\partial t}=0 \quad \text { for } x=0, i=0,1, \ldots, \\
\frac{\partial^{2} \alpha_{10}}{\partial t^{2}}+K_{r} \frac{1}{a_{0}} \frac{\partial \alpha_{10}}{\partial x}=0 \quad \text { for } x=1 \\
\frac{\partial^{2} \alpha_{11}}{\partial t^{2}}+K_{r} \frac{1}{a_{0}} \frac{\partial \alpha_{11}}{\partial x}=-\frac{1}{a_{c}} \frac{\partial^{2} \alpha_{10}}{\partial t^{2}} \sum_{m=1}^{\infty}\left(a_{m} \cos m \Omega_{0} t+b_{m} \sin m \Omega_{0} t\right) \\
+\frac{1}{a_{c}} \Omega_{0} \frac{\partial \alpha_{10}}{\partial t} \sum_{m=1}^{\infty} m\left(a_{m} \sin m \Omega_{0} t-b_{m} \cos m \Omega_{0} t\right) \\
+\frac{1}{2} \Omega_{0}^{2} \frac{1}{a_{c}} \alpha_{10} \sum_{m=1}^{\infty} m^{2}\left(a_{m} \cos m \Omega_{0} t+b_{m} \sin m \Omega_{0} t\right) \\
+\frac{1}{2 a_{c}} \Omega_{0}^{2} \sum_{m=1}^{\infty} m\left(a_{m} \sin m \Omega_{0} t-b_{m} \cos m \Omega_{0} t\right) \quad \text { for } x=1 .
\end{gathered}
$$

Analogously appropriate relations for $\alpha_{12}, \alpha_{13}, \ldots$ in $x=1$ can be derived.

We are interested in a forced response, so $\alpha_{10}(x, t)=0$, and the solution for $\alpha_{11}$, according to (3.8), is sought in the form

$$
\alpha_{11}(x, t)=\sum_{m=1}^{\infty}\left(A_{11 m} \sin m \Omega_{0} t+B_{11 m} \cos m \Omega_{0} t\right) \sin m \Omega_{0} x .
$$

This solution satisfies the relation for $x=0$, and from the relation for $x=1$ the amplitudes $A_{11 m}$ and $B_{11 m}$ are determined. They are equal correspondingly to

$$
\begin{gathered}
A_{11 m}=\frac{1}{2 a_{c}} \cdot \frac{\Omega_{0} a_{m}}{-\beta_{m} \sin \beta_{m}+K_{r}\left(a_{0}\right)^{-1} \cos \beta_{m}}, \\
B_{11 m}=-\frac{1}{2 a_{c}} \cdot \frac{\Omega_{0} b_{m}}{-\beta_{m} \sin \beta_{m}+K_{r}\left(a_{0}\right)^{-1} \cos \beta_{m}},
\end{gathered}
$$

where $\beta_{m}=m \Omega_{0}$. The above relations are the same as in [1] and they can be used, apart from (2.11) with $N=1$, in numerical calculations for the system with a single elastic element. In multimass systems, (2.11) with a retarded argument and with variable coefficients are proposed to be applied.

\section{Numerical results}

In the beginning, numerical calculations are performed for a two-mass system using (2.11) with $N=1$ and $K_{r}=0.05$. The mass moment of inertia of the rigid body 2 is 


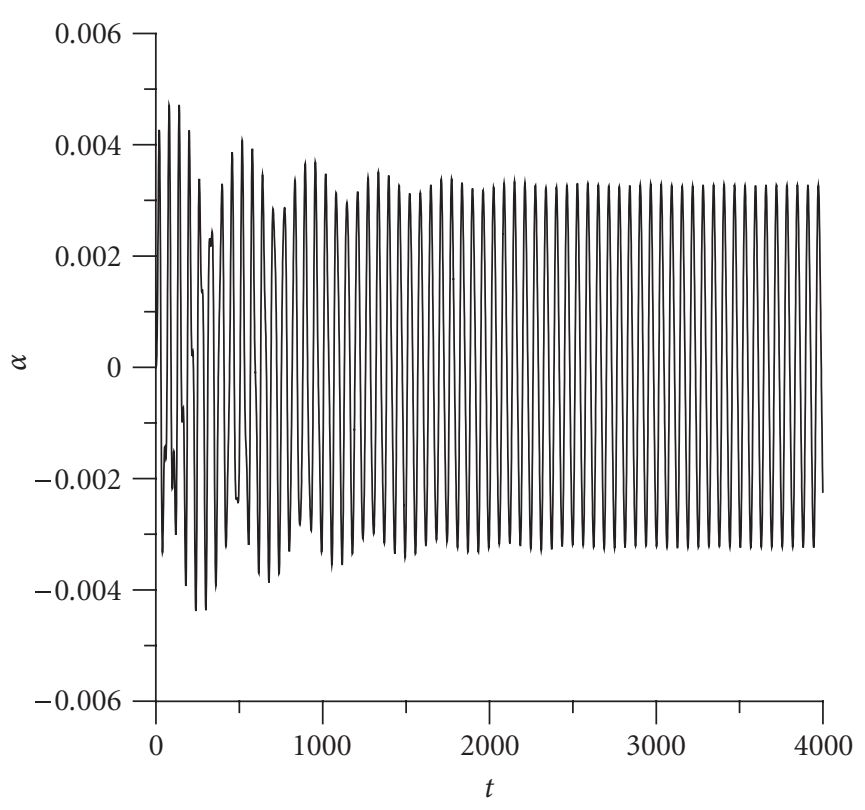

Figure 4.1. Displacements in $x=1$.

assumed in the form [1],

$$
J_{2}\left(\Omega_{0} t\right)=a_{0}+a_{2} \cos \left(2 \Omega_{0} t\right)
$$

where $a_{0}=1$. According to the Fourier series (3.3), in formula (4.1) only two terms are being taken into account, namely, with $m=0$ and $m=2$.

In Figures 4.1-4.4 numerical results for angular displacements and velocities in crosssections $x=1$ and $x=0.5$ for $t<4000.0$ are plotted with $a_{2}=0.05$ and $\Omega_{0}=0.05$ with zero initial conditions. The diagrams show the results in transient and in the steady states. It is seen that displacements as well as velocities are smaller in the cross-section $x=0.5$.

Now, numerical and analytical results are compared in a steady state. For the mass moment of inertia $J_{2}$ expressed by (4.1) the approximate solution is $\alpha_{1}(x, t)=\varepsilon \alpha_{11}(x, t)$, where according to (3.4) and (3.9)

$$
\alpha_{11}(x, t)=A_{112} \sin 2 \Omega_{0} x \sin 2 \Omega_{0} t
$$

and $A_{112}$ is determined by (3.10). This solution is a harmonic function. That is seen in Figure 4.5. Moreover, from Figure 4.5 it follows that numerical and analytical solutions are practically the same in a steady state.

Diagrams in Figures 4.1-4.4 inform that numerical solutions achieve steady states after rather long intervals of time because damping is neglected in the considered systems. For this reason, amplitude-frequency curves for angular displacements in the cross-section 
8 Modeling of systems with variable inertia

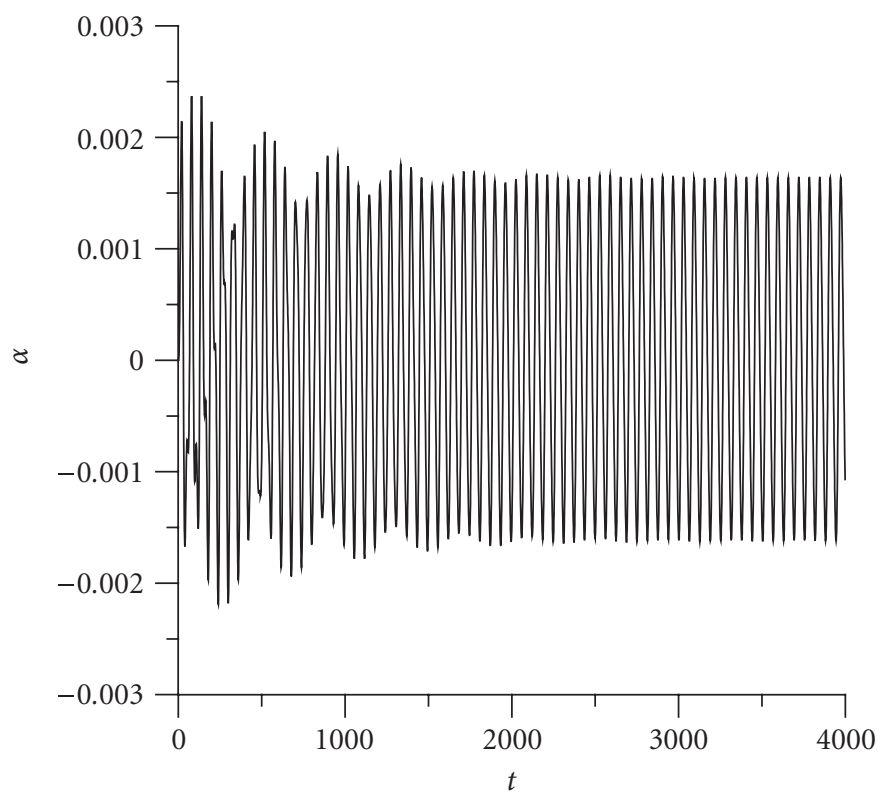

Figure 4.2. Displacements in $x=0.5$.

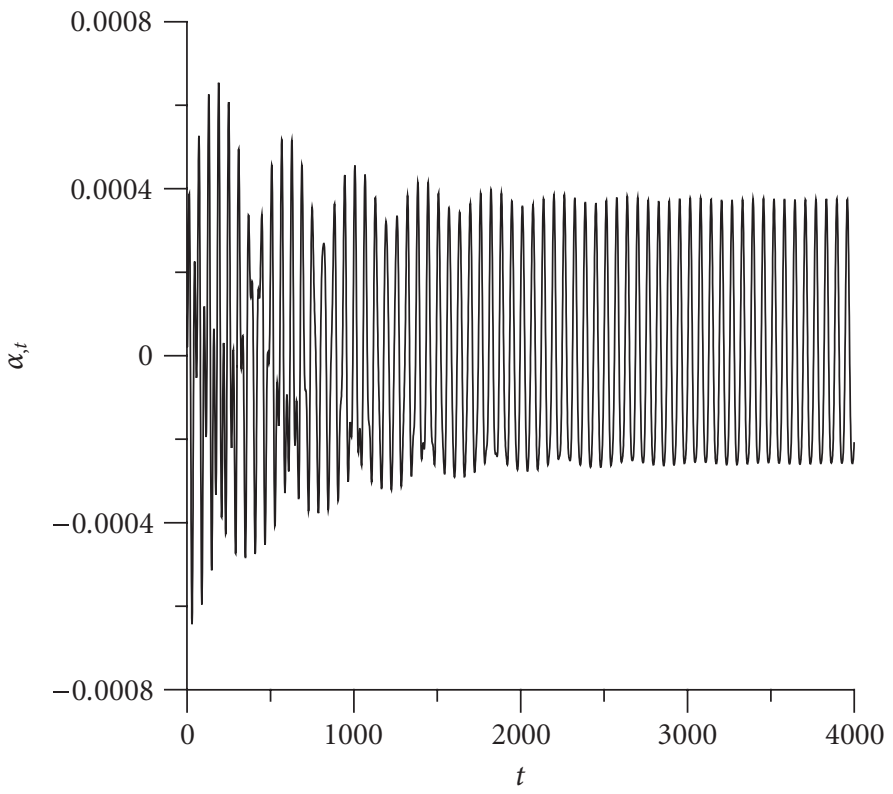

Figure 4.3. Velocities in $x=1$. 


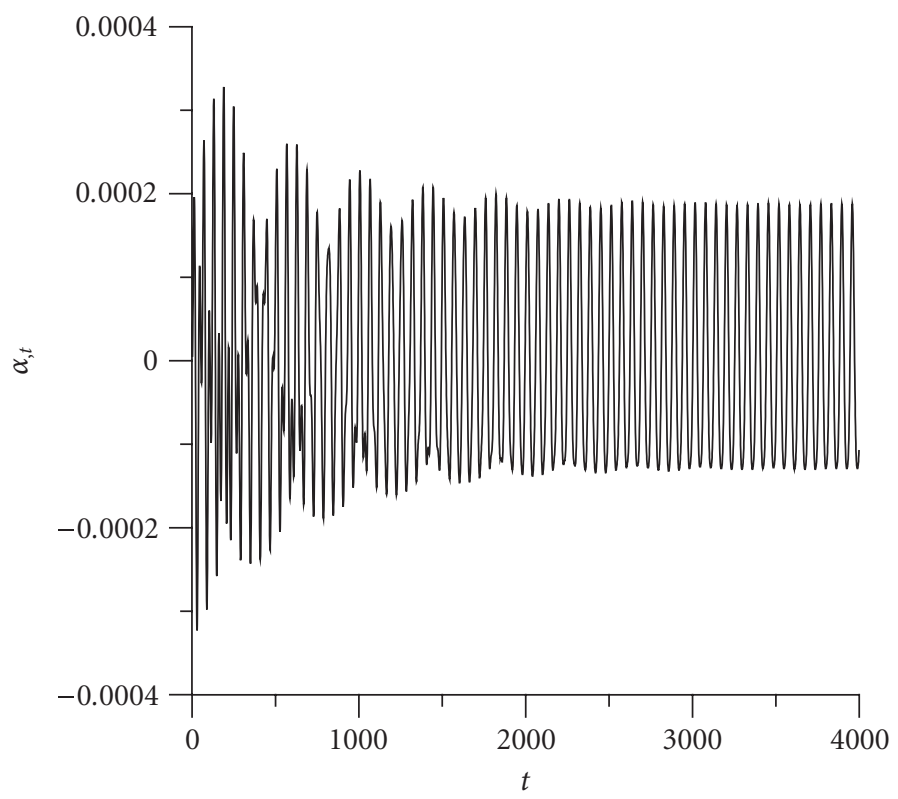

Figure 4.4. Velocities in $x=0.5$.

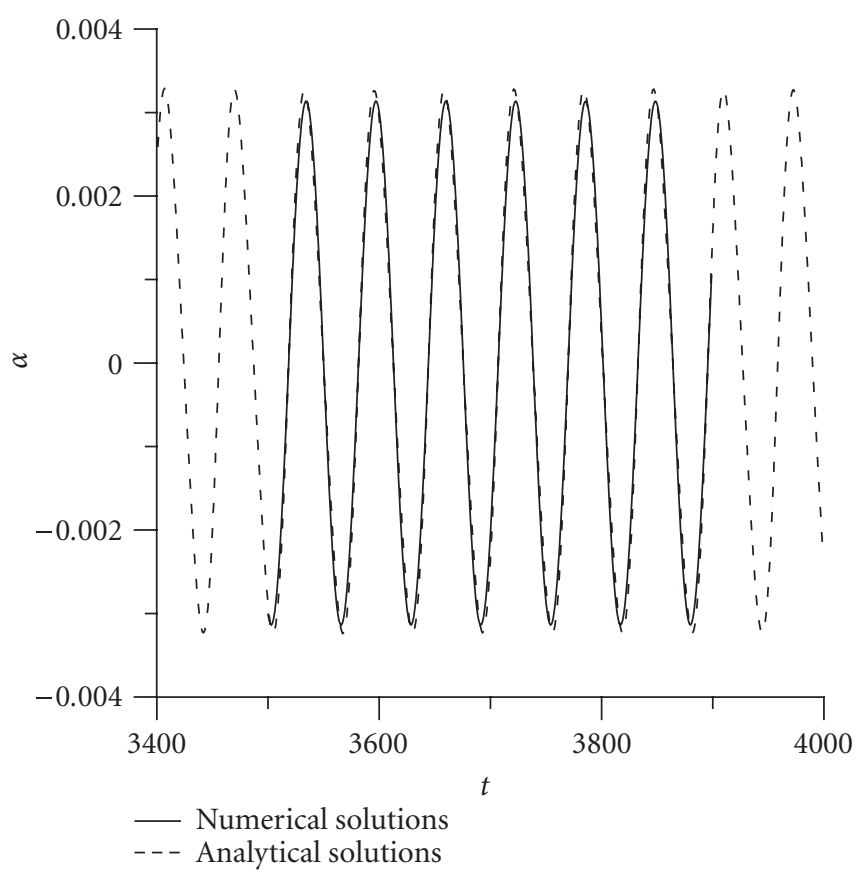

Figure 4.5. Numerical and analytical solutions for displacements in $x=1$ in a steady state. 


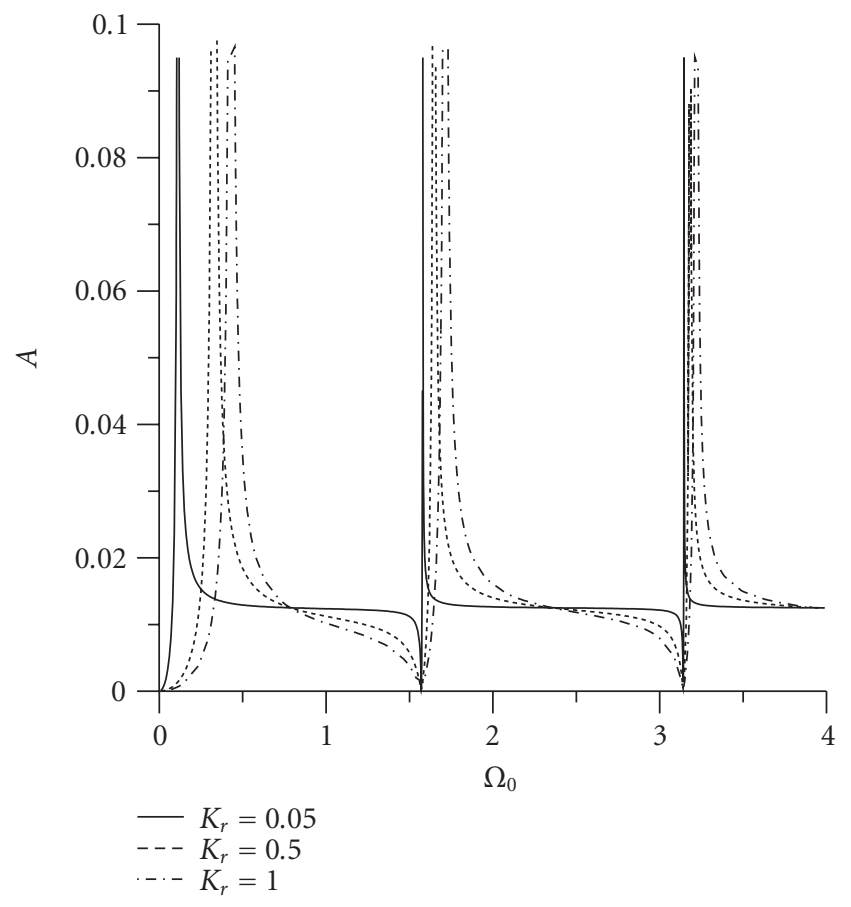

Figure 4.6. Amplitude-frequency curves according to the analytical solution (4.2) for $K_{r}=0.05, K_{r}=$ 0.5 , and $K_{r}=1.0$.

$x=1$ are presented using only the analytical solution. They are shown in Figure 4.6 for $K_{r}=0.05, .5,1.0$ and $\Omega_{0}<4.0$. The diagrams contain three resonant regions. The amplitudes of the analytical solution tend to infinity for $\Omega_{0}=\omega_{1} / 2, \Omega_{0}=\omega_{2} / 2$ and $\Omega_{0}=\omega_{3} / 2$, where $\omega_{i}$ are the first three natural frequencies of the considered systems. From Figure 4.6 it follows that the effect of $K_{r}$ is observed mainly in the first resonant regions.

\section{Conclusions}

From the considerations presented in the paper it follows that the problem of the torsional two-mass system with variable inertia discussed in [1] can be generalized to a multimass discrete-continuous system in such a way that the wave method can be applied in the study. An analytical solution in a special case is derived. The analytical approach and numerical solution for $N=1$ give comparable results only in steady states.

\section{References}

[1] K. Koser and F. Pasin, Continuous modelling of the torsional vibrations of the drive shaft of mechanisms, Journal of Sound and Vibration 188 (1995), no. 1, 17-24.

[2] W. Nadolski and A. Pielorz, Theoretical investigations of nonlinear loads on gear teeth in single gear transmission, International Journal of Mechanical Sciences 43 (2001), no. 2, 299-311. 
[3] M. S. Pasricha and W. D. Carnegie, Formulation of the equations of dynamic motion including the effects of variable inertia on the torsional vibrations in reciprocating engines, part $I$, Journal of Sound and Vibration 66 (1979), no. 2, 181-186.

[4] A. Pielorz, Dynamic analysis of a nonlinear discrete-continuous torsional system by means of wave method, Zeitschrift für Angewandte Mathematik und Mechanik 75 (1995), no. 9, 691-698.

[5] __ Non-linear vibrations of a discrete-continuous torsional system with non-linearities having characteristic of a soft type, Journal of Sound and Vibration 225 (1999), no. 2, 375-389.

[6] S. C. Sinha and E. A. Butcher, Symbolic computation of fundamental solution matrices for timeperiodic dynamical systems, Journal of Sound and Vibration 206 (1997), no. 1, 61-85.

[7] J. Zajączkowski, Torsional vibration of shafts coupled by mechanisms, Journal of Sound and Vibration 116 (1987), no. 2, 221-237.

Amalia Pielorz: Faculty of Management and Computer Modelling, Kielce University of Technology, Aleja 1000-lecia Pañstwa Polskiego 7, 25-314 Kielce, Poland

E-mail address: apielorz@tu.kielce.pl

Monika Skóra: Faculty of Management and Computer Modelling, Kielce University of Technology, Aleja 1000-lecia Pañstwa Polskiego 7, 25-314 Kielce, Poland

E-mail address: mskora@tu.kielce.pl 


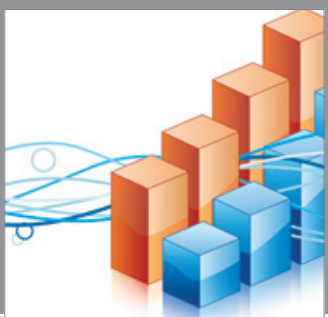

Advances in

Operations Research

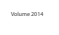

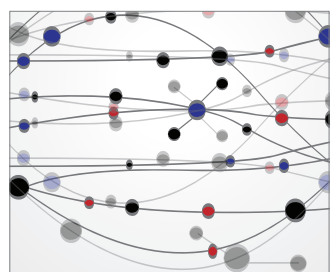

\section{The Scientific} World Journal
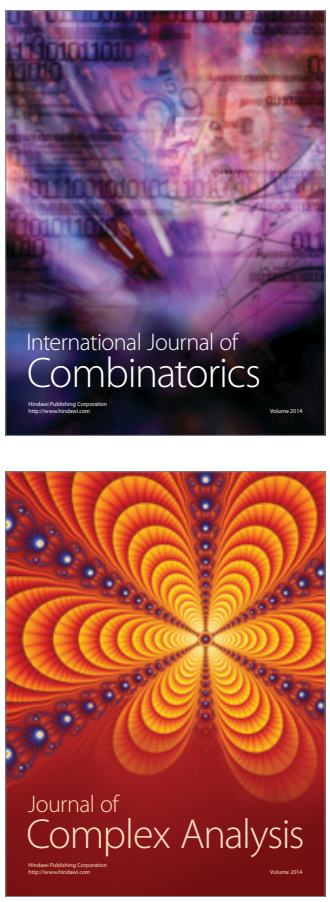

International Journal of

Mathematics and

Mathematical

Sciences
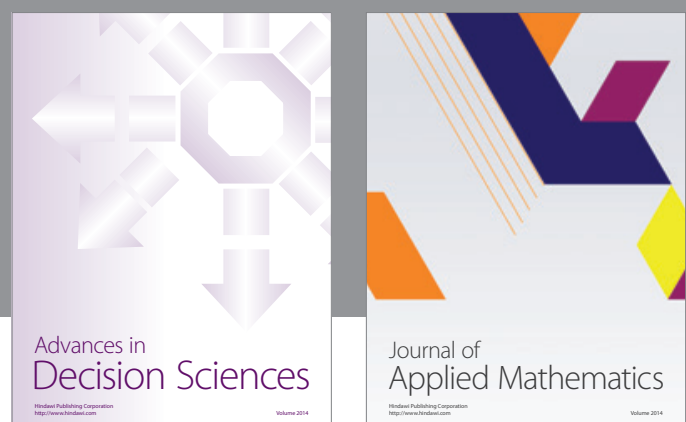

Journal of

Applied Mathematics
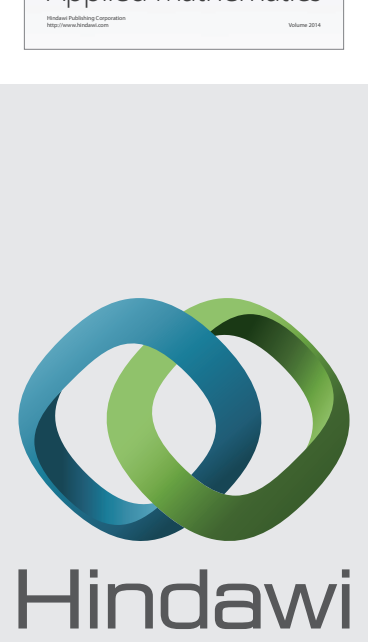

Submit your manuscripts at http://www.hindawi.com
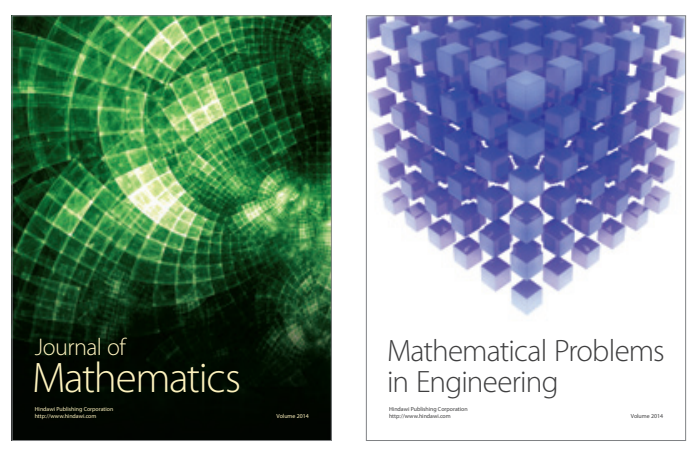

Mathematical Problems in Engineering
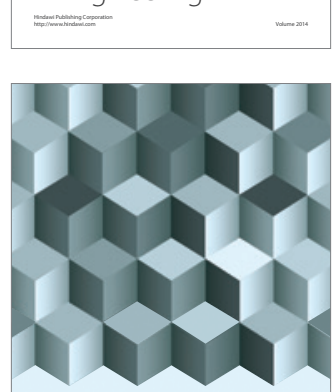

Journal of

Function Spaces
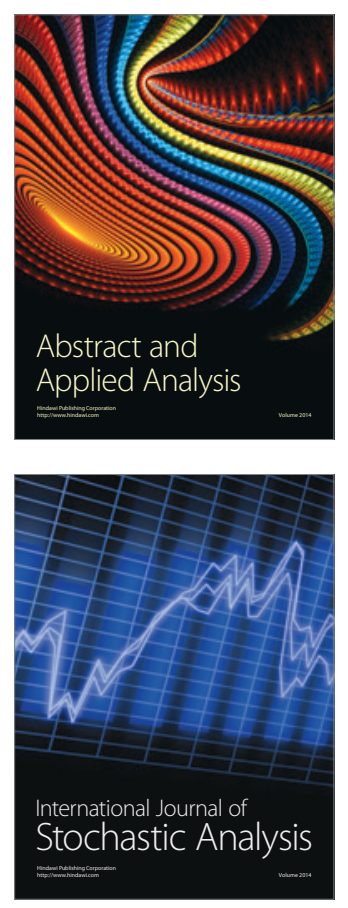

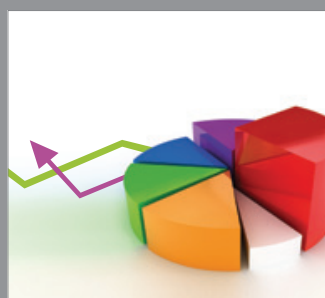

ournal of

Probability and Statistics

Promensencen
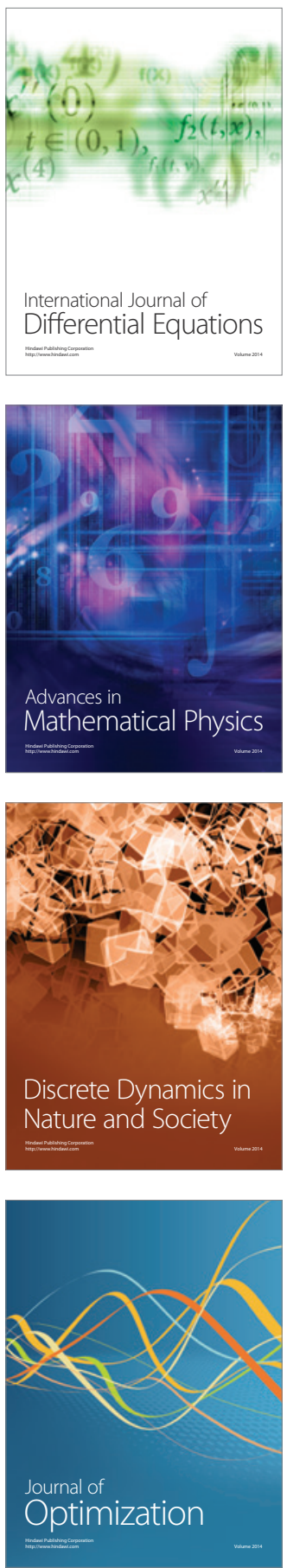